\title{
COCHRANE CORNER Systematic reviews of bicycle helmet research
}

$\checkmark r$

he recently published systematic review of the effectiveness of bicycle helmet legislation brings to a total of three published reviews on this topic in the Cochrane Database of Systematic Reviews:

1. Helmets for preventing head and facial injuries in bicyclists; ${ }^{1}$

2. Non-legislative interventions for the promotion of cycle helmet wearing by children; ${ }^{2}$ and

3. Bicycle helmet legislation for the uptake of helmet use and prevention of head injuries. ${ }^{3}$

Cycling is a cheap and efficient form of transport in many low and middle income countries, and is a common recreational activity in high income countries. With current concerns about global warming, the popularity of cycling, with both cardiovascular and environmental benefits, is likely to rise. However, bicycle-related head injury is a significant contributor to hospitalisations and death worldwide.

It seems intuitive that bicycle helmets would be an effective means of reducing head injury, and indeed helmet manufacturers and standards associations worldwide conduct rigorous tests to determine the impact absorption and other qualities of helmets. It is however, important to go beyond laboratory tests and understand whether helmets reduce injuries in the event of a crash in real-life; hence the review by Thompson et al. ${ }^{1}$ This review included five well conducted casecontrol studies and found that helmets provide a $63-88 \%$ reduction in the risk of head, brain and severe brain injury for all ages of bicyclists. Helmets were found to provide equal levels of protection for crashes involving motor vehicles (69\%) and crashes from all other causes (68\%). Furthermore, injuries to the upper and mid facial areas were found to be reduced by $65 \%$, although helmets did not prevent lower facial injuries. The review authors concluded that bicycle helmets are an effective means of preventing head injury.

However, despite the evidence of the efficacy of helmets in preventing serious injury, they are not universally used, even where legislation requiring their use is introduced. As a result there have been various attempts to promote use of helmets in school age children via social marketing and education campaigns. The review by Royal et $a l^{2}$ examined the effectiveness of such non-legislative interventions on cycle helmet wearing by children. These included health education programs, subsidised or free helmet distribution programs, media campaigns, or interventions that included elements of the above.
This review included 22 studies, including three individually randomised controlled trials, six cluster randomised controlled trials, 12 controlled before-after studies and one trial with concurrent controls; random-effects models were used to pool data from individual studies in meta-analyses. In general, the programs seemed to be effective (OR 2.30, 95\% CI 1.37 to 3.85 ), with a greater effect for community-based studies and those providing free helmets than for those providing subsidised helmets and for those set in schools. None of the studies examined the impact of the program on injury rates. No adverse events were recorded but the review authors' caution that it is possible that interventions to promote cycle helmet wearing may reduce cycling, with negative health effects.

Bicycle helmet legislation is another important intervention introduced in many high income countries to increase helmet wearing. The systematic review by Macpherson and Spinks, ${ }^{3}$ published in 2007, examined the effectiveness of bicycle helmet legislation on bicycle-related injury and helmet use. To ensure that other environmental and legislative changes including changes in cycling rates, improved bicycle paths and lower vehicle speeding limits were taken into account, this review had stringent methodologic criteria to ensure that only studies with a concurrent control group were included. Only three of the five controlled time series analyses included in the review examined the impact of helmet legislation on head injuries, with two finding a significant protective effect. The third study reported a non-significant decline in the proportion of head injuries compared with other bicycle-related trauma. Helmet use was found to increase significantly from between $45 \%$ and $84 \%$ with the introduction or enforcement of helmet legislation.

None of the studies included in the review measured pre- and post-legislation cycling participation rates, and so it was not possible to comment on the potential adverse effect of helmet legislation. The review authors concluded that based on the best available evidence, there appeared to be a protective effect of bicycle helmet legislation against head injury among cyclists, but that that there was an important need for future evaluative research to include appropriately concurrent control groups, to measure actual helmet use, and to record cycling participation rates.

So where to from here? There is good evidence that bicycle helmets are effective in reducing head and facial injury in the event of a crash, and that helmet legislation is also likely to be effective at a population level, although high quality controlled research must continue. We know that non-legislative interventions are effective in increasing helmet wearing rates in children, particularly communitybased programs that provide free helmets.

However, there is a steep socioeconomic gradient for injuries to cyclists, with higher mortality rates for children from deprived backgrounds; ${ }^{4}$ yet the review by Royal et al was not able to identify the best way to increase wearing rates in this population. Further research that addresses such questions is clearly important.

Furthermore, cyclists are also vulnerable road users at increased risk in low and middle income countries: in Delhi, India, cyclists constitute $5 \%$ of the trips but $14 \%$ of total road traffic injury related deaths, ${ }^{5}$ but almost all of the research on bicycle helmets has been conducted in high income countries. It seems likely, given its potential cost effectiveness as an intervention to reduce head injury, that helmet legislation may also useful in low and middle income countries where cycling is common. Although it seems plausible that helmets would work equally effectively in such settings, there is a need for high quality controlled intervention studies examining both effectiveness in relation to head injury but also effective mechanisms for increasing wearing rates, including management of helmet quality and supply and issues relating to enforcement.

For further information about the Cochrane Collaboration visit http:// cochrane.org or, for information about the Cochrane Injuries Group, email katharine. ker@lshtm.ac.uk. The Cochrane Collaboration offers training at centers worldwide for potential review authors-see the website for details.

\section{Rebecca Ivers, \\ External Editor, Cochrane Injuries Group rivers@george.org.au}

\section{REFERENCES}

1 Thompson DC, Rivara FP, Thompson R. Helmets for preventing head and facial injuries in bicyclists. Cochrane Database Syst Rev 1999:4:CD001855.

2 Royal ST, Kendrick D, Coleman T. Non-legislative interventions for the promotion of cycle helmet wearing by children. Cochrane Database Syst Rev 2005;2:CD003985.

3 Macpherson A, Spinks A. Bicycle helmet legislation for the uptake of helmet use and prevention of head injuries. Cochrane Database Syst Rev 2007; 2:CD005401.

4 Roberts I. Cause specific social class mortality differentials for child injury and poisoning in England and Wales. J Epidemiol Community Health 1997;51:334-5.

5 Mohan D. Work trips and safety of bicyclists. Indian Journal of Transport Management 2002;26:225-33. 